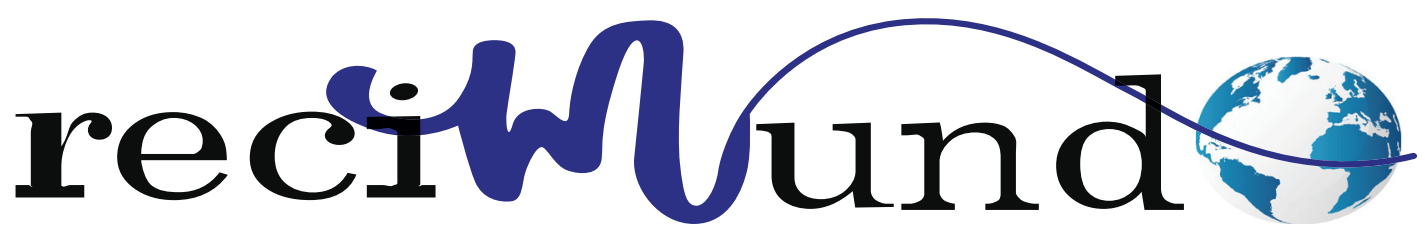

Revista Científica Mundo de la Investigación y el Conocimiento

DOI: 10.26820/recimundo/5.(2).abril.2021.179-186

URL: https://recimundo.com/index.php/es/article/view/1052

EDITORIAL: Saberes del Conocimiento

REVISTA: RECIMUNDO

ISSN: 2588-073X

TIPO DE INVESTIGACIÓN: Artículo de revisión

Código UNESCO: 32 Ciencias Médicas

PAGINAS: $179-186$

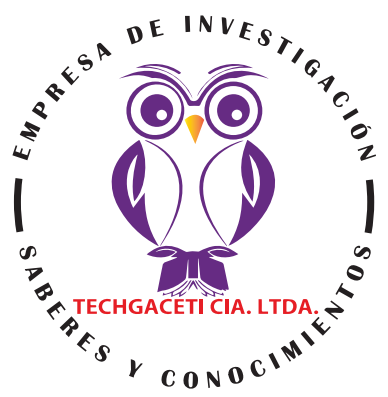

\title{
Manejo de emergencia en intoxicación por plaguicidas
}

Emergency management in pesticide poisoning

Características clínicas da síndrome HELLP

Armando Alberto Díaz Pérez'; Jessenia Marcela Luzuriaga Amador²; María Fernanda Monroy Pesantez; Juan Antonio Vera Hinojosa ${ }^{4}$

RECIBIDO: 15/01/2021 ACEPTADO: 20/03/2021 PUBLICADO: 01/04/2021

1. Médico General; Hospital General Milagro; Milagro, Ecuador; armandocs11@hotmail.com; (iD https://orcid.org/0000-0002$7520-4855$

2. Magister en Negocios Internacionales y Gestión de Comercio Exterior; Especialista en Negocios Internacionales; Diploma Superior en Comercio Exterior; Contadora Publica Autorizada; Investigadora Independiente; Milagro, Ecuador; marlaluzuriaga@hotmail.com; iD https://orcid.org/0000-0001-7717-6088

3. Médico General en Funciones Hospitalarias; Hospital General Guasmo Sur; Guayaquil, Ecuador; mafer.monroy309@gmail. com; (iD) https://orcid.org/0000-0003-1362-4886

4. Médico General; Residente Hospital General Milagro; Ecuador; juanantonioverahinojosa@gmail.com; (iD https://orcid. org/0000-0002-1904-8595

CORRESPONDENCIA

Armando Alberto Díaz Pérez

armandocs11@hotmail.com

Milagro, Ecuador

() RECIMUNDO; Editorial Saberes del Conocimiento, 2021 


\section{RESUMEN}

El uso de los plaguicidas ha producido grandes beneficios agrícolas y, a la vez, graves problemas de salud pública que requieren solución. Para destacar la importancia económica de la industria de los plaguicidas en el mundo, basta señalar que las ventas mundiales de las 20 principales compañías productoras sobrepasaron los tres millones de toneladas y que su facturación ascendió a US\$21000 millones a inicios de la presente década. Numerosos trabajos han demostrado la producción de intoxicaciones agudas por plaguicidas en seres humanos en diversos lugares del mundo. Calculan algunos estudios que el número anual de intoxicaciones agudas por plaguicidas oscila entre 500000 y 1528000 y que cada año se producen de 3000 a 28000 defunciones por esa causa. En las estadísticas se indica que las intoxicaciones constituyen el 2.5 al $3 \%$ de los casos atendidos en emergencia. Y del total de pacientes intoxicados el $28 \%$ corresponde a organofosforados y de estos el $15 \%$ fallecen por no tener acceso inmediato a un área de salud y recibir atención oportuna. La metodología de la investigación, es una revisión bibliográfica, la cual se ha apoyado en medios electrónicos como Google Académico, PubMed, entre otros, en donde se ha encontrado información de diferentes trabajos de investigación, que aborda la temática aquí planteada. Ente algunas de las conclusiones destacan que una intoxicación por plaguicida puede ser mortal, independientemente de por cuál vía la persona haya tenido contacto con el mismo. Entre algunos de los síntomas que se pueden presentar, son dificultad respiratoria, disnea, irritación en ojos y piel, bradicardia, entre otros. Es por ello que la valoración inicial que se haga en el centro de salud por el personal médico más la información que pueda proporcionar los familiares, compañeros de trabajo, del intoxicado acerca del tipo de plaguicida puede ser determinante para el abordaje rápido en cuanto a la administración del tratamiento más adecuado. El tratamiento va a depender del grado de la intoxicación, sin embargo, entre los tipos de tratamiento está el uso de Atropina y Oximinas, colocación de sondas nasogástricas y lavado gástricos, administración de carbón activado, entre otros.

Palabras clave: Intoxicación, Plaguicidas, Organofosforados, Agrícola, Tratamiento.

\section{ABSTRACT}

The use of pesticides has produced great agricultural benefits and, at the same time, serious public health problems that require solution. To highlight the economic importance of the pesticide industry in the world, it is enough to point out that the global sales of the 20 main producing companies exceeded three million tons and that their turnover amounted to US $\$$ 21 billion at the beginning of this decade. Numerous studies have demonstrated the production of acute pesticide poisoning in humans in various parts of the world. Some studies estimate that the annual number of acute pesticide poisonings ranges between 500,000 and 1,528,000 and that each year there are 3,000 to 28,000 deaths from this cause. The statistics indicate that poisonings constitute 2.5 to $3 \%$ of the cases treated in emergencies. And of the total of intoxicated patients, $28 \%$ correspond to organophosphates and of these 15\% die because they do not have immediate access to a health area and receive timely care. The research methodology is a bibliographic review, which has been supported by electronic media such as Google Scholar, PubMed, among others, where information from different research works has been found, which addresses the issue raised here. Among some of the conclusions they highlight that pesticide poisoning can be fatal, regardless of which way the person has had contact with it. Some of the symptoms that may occur are respiratory distress, dyspnea, eye and skin irritation, bradycardia, among others. That is why the initial assessment made in the health center by the medical staff plus the information that the family members, co-workers, of the intoxicated person about the type of pesticide can provide can be decisive for the rapid approach regarding the administration of the most appropriate treatment. Treatment will depend on the degree of intoxication, however, among the types of treatment is the use of Atropine and Oximins, placement of nasogastric tubes and gastric lavage, administration of activated charcoal, among others.

Keywords: Intoxication, Pesticides, Organophosphates, Agricultural, Treatment.

\section{RESUMO}

O uso de pesticidas tem produzido grandes benefícios agrícolas e, ao mesmo tempo, graves problemas de saúde pública que requerem solução. Para destacar a importância econômica da indústria de pesticidas no mundo, basta apontar que as vendas globais das 20 principais empresas produtoras ultrapassaram três milhões de toneladas e que seu faturamento foi de US\$21 bilhões no início desta década. Numerosos estudos têm demonstrado a produção de intoxicações agudas por pesticidas em humanos em várias partes do mundo. Alguns estudos estimam que o número anual de intoxicações agudas por pesticidas varia entre 500.000 e 1.528 .000 e que a cada ano há de 3.000 a 28.000 mortes por esta causa. As estatísticas indicam que as intoxicações constituem 2,5 a 3\% dos casos tratados em situações de emergência. E do total de pacientes intoxicados, $28 \%$ correspondem a organofosforados e destes $15 \%$ morrem por não terem acesso imediato a uma área de saúde e receberem atendimento em tempo hábil. A metodologia de pesquisa é uma revisão bibliográfica, que tem sido apoiada por meios eletrônicos como o Google Scholar, PubMed, entre outros, onde foram encontradas informações de diferentes trabalhos de pesquisa, que abordam a questão aqui levantada. Entre algumas das conclusões destacam que o envenenamento por pesticidas pode ser fatal, independentemente de como a pessoa tenha tido contato com ele. Alguns dos sintomas que podem ocorrer são angústia respiratória, dispnéia, irritação dos olhos e da pele, bradicardia, entre outros. É por isso que a avaliação inicial feita no centro de saúde pelo pessoal médico mais as informações que os membros da família, colegas de trabalho, da pessoa intoxicada sobre o tipo de pesticida podem fornecer pode ser decisiva para a rápida abordagem em relação à administração do tratamento mais apropriado. O tratamento dependerá do grau de intoxicação, entretanto, entre os tipos de tratamento está o uso de Atropina e Oximinas, colocação de tubos nasogástricos e lavagem gástrica, administração de carvão ativado, entre outros.

Palavras-chave: Intoxicação, Pesticidas, Organofosfatos, Agricultura, Tratamento. 


\section{Introducción}

En las estadísticas se indica que las intoxicaciones constituyen el 2.5 al $3 \%$ de los casos atendidos en emergencia. Y del total de pacientes intoxicados el $28 \%$ corresponde a organofosforados y de estos el $15 \%$ fallecen por no tener acceso inmediato a un área de salud y recibir atención oportuna (Castro Valencia, 2015, pág. 4).

La utilización de sustancias tóxicas en la agricultura conlleva riesgos en la salud de los trabajadores agrícolas, principalmente de quienes las manipulan, así como, para el medio ambiente, por la contaminación de suelos, aire y agua. El uso indiscriminado de plaguicidas sin las precauciones debidas, potencia los riesgos de intoxicación aguda y crónica a los trabajadores, principalmente los jornaleros o peones agrícolas. También, existe el riesgo de residualidad en los alimentos, que es igualmente peligroso para la población general. En sociedades agrícolas donde hay poca o nula vigilancia epidemiológica y de las normas del uso y manejo de plaguicidas, es frecuente que en diversos reportes y noticias en los medios de comunicación se aluda a la muerte o gravedad de la salud de peones intoxicados por estas sustancias, sin mayor detenimiento que la noticia misma (Conejo, Órnelas, \& de los Ángeles, s.f).
Los casos de intoxicaciones por plaguicidas, se han agrupado en:

- Intoxicaciones de origen laboral: Intoxicación que se producen a causa o con ocasión de su trabajo (temporeros, fumigadores, tractoristas, mezcladores, etc.) sea en el lugar del trabajo o en relación con las tareas que desempeña.

- Intoxicaciones de origen voluntario: se producen cuando la persona por iniciativa propia, y consciente de sus actos, ingiere, huele o se impregna de uno o varios plaguicidas, con fines suicidas.

- Intoxicaciones no laborales o accidentales: aquellas producidas por una causa distinta a la laboral ej. aplicación de plaguicidas en la comunidad o sector; o bien cuando por error se hace contacto con un plaguicida.

- Intoxicaciones provocadas: aquellas intoxicaciones producidas a una persona por agresión de un tercero, con el fin de causarle daño (Gutiérrez, 2010).

\section{Clasificación toxicológica de los plagui- cidas}

Los plaguicidas se clasifican toxicológicamente en tres categorías, las cuales pueden ser identificadas por el color de la etiqueta y la presentación.

Tabla 1. Clasificación toxicológica de los plaguicidas

\begin{tabular}{|c|c|c|c|c|c|}
\hline Categoría & \multicolumn{5}{|c|}{ LD 50 Aguda (ratas) mg/kg. } \\
\hline \multirow[t]{2}{*}{ Toxico } & \multicolumn{2}{|c|}{ Oral } & \multicolumn{2}{|c|}{ Dermal } & Color \\
\hline & Sólido & Líquido & Sólido & Líquido & Etiqueta \\
\hline 1 Extremamente & $<5$ & $>20$ & $>10$ & $>40$ & ROJA \\
\hline 2 Altamente & $<5$ & $>20$ & $>10$ & $>40$ & AMARILLA \\
\hline 3 Moderadamente & $>50$ & $>200$ & $>100$ & $>400$ & AZUL \\
\hline 4 Ligeramente & $>500$ & $>2000$ & $>1000$ & $>4000$ & VERDE \\
\hline
\end{tabular}

Fuente: (Aveiga Valdivieso, 2020) 
El uso de los plaguicidas ha producido grandes beneficios agrícolas y, a la vez, graves problemas de salud pública que requieren solución. Para destacar la importancia económica de la industria de los plaguicidas en el mundo, basta señalar que las ventas mundiales de las 20 principales compañías productoras sobrepasaron los tres millones de toneladas y que su facturación ascendió a US\$21000 millones a inicios de la presente década. Numerosos trabajos han demostrado la producción de intoxicaciones agudas por plaguicidas en seres humanos en diversos lugares del mundo. Calculan algunos estudios que el número anual de intoxicaciones agudas por plaguicidas oscila entre 500000 y 1528000 y que cada año se producen de 3000 a 28000 defunciones por esa causa (Aveiga Valdivieso, 2020, pág. 13).

Las vías de entrada del tóxico son la digestiva (intentos de suicidio o exposición accidental), la cutánea y/o la inhalatoria (exposición accidental en el ámbito laboral), en tanto que es excepcional la intoxicación parenteral (Barco, y otros, 2008, pág. 207). Es preciso recordar que los signos y síntomas de intoxicación aguda aparecen inmediatamente o en un lapso de 1/2 - 1 hora después de la exposición al tóxico, se tiene pues, que el organofosforado causa la perdida de la función enzimática, lo que permite la acumulación de la acetilcolina, impidiendo la transmisión normal de los impulsos desde las fibras nerviosas hasta los tejidos inervados. De allí pues que se producen ciertas manifestaciones clínicas, las cuales dependen del tipo de receptor donde actúe el tóxico (Cuasapaz Guama, 2017).

Dentro de las complicaciones más frecuentemente observadas en los pacientes atendidos por intoxicación con pesticidas se encuentran aquellas asociadas al sistema respiratorio (insuficiencia respiratoria), cardiovascular (arritmias e hipotensión), renales (insuficiencia renal aguda y su- baguda), afecciones gastrointestinales y neurológicas (convulsiones). Siendo éstas las responsables del importante índice de mortalidad asociado a la ingesta de estas sustancias (Cerna Urrutia \& Trigueros Jovel, 2015, pág. 3).

\section{Metodología}

La metodología de la investigación, es una revisión bibliográfica, la cual se ha apoyado en medios electrónicos como Google Académico, PubMed, entre otros, en donde se ha encontrado información de diferentes trabajos de investigación, que aborda la temática aquí planteada. El objetivo fundamental de esta investigación, es generar un documento que sintetice todos los aspectos relevantes sobre la intoxicación por plaguicidas y que sea un punto referencia para futuras investigaciones.

\section{Resultados}

Las intoxicaciones por plaguicidas responden a numerosos tóxicos de las cuales las más características por su frecuencia son:

- Organofosforados: Son los insecticidas más usados actualmente. Se utilizan en jardines, en el hogar, en agricultura y en la práctica veterinaria. Son muy tóxicos. Algunos de los más empleados son: Malatión, paratión (muy tóxico), fentión, diclorvós, menvinfós, etc.

- Son absorbidos por inhalación, por ingestión o por vía cutánea.

- Carbamatos: Carbaril, aldicarb, propoxur, metiocarb, etc. Estos insecticidas son muy utilizados en el hogar, jardín y agricultura. La combinación carbamilo-acetilcolinesterasa se disocia más rápidamente que el complejo producido por los organofosforados. Esto hace que el intervalo entre la dosis que produce los síntomas y la dosis letal sea mayor que el de los organofosforados, y que la medición de la actividad de colinesterasa en sangre como indicador diagnós- 
tico no sea valorable. Se absorben por inhalación, ingesta y por la piel.

- Organoclorados sólidos: Endosulfán, dienoclor, indano, hexaclorociclohexano, lindano, etc.Algunos de ellos (DDT, aldrín, endrín, clordano) están retirados en muchos países. La intoxicación se produce por ingesta, inhalación o a través de la piel. Su dosis tóxica es de 3-3,5 g.

- Paraquat y diquat: Son herbicidas bipiridilos. Afectan al tracto gastrointestinal, riñón, hígado, corazón y otros órganos. El paraquat causa fibrosis pulmonar, manos secas y agrietadas y caída de las uñas. La ingestión produce sensación de quemadura en la boca, garganta, tórax y abdomen, edema pulmonar, pancreatitis, afectación renal y del SNC. El diquat produce toxicidad neurológica (Aveiga Valdivieso, 2020).

- Principios generales en el manejo de los envenenamientos agudos causados por pesticidas.

Inicialmente se debe proceder al reconocimiento de la gravedad de la intoxicación, y de situaciones que pongan en peligro la vida del paciente, para lo cual es necesario determinar las funciones vitales y considerar a las intoxicaciones agudas como un trauma múltiple de origen químico, porque permite aplicar las técnicas básicas y avanzadas de apoyo vital, en el manejo inicial del paciente a su ingreso a los servicios de urgencias (Aveiga Valdivieso, 2020).

Tabla 2. Manejo de intoxicación

\begin{tabular}{|c|c|}
\hline $\begin{array}{l}\text { Vigile las vías } \\
\text { respiratorias. }\end{array}$ & Garantizar permeabilidad de las vías respiratorias. \\
\hline $\begin{array}{c}\text { Canalizar vía } \\
\text { venosa periférica }\end{array}$ & $\begin{array}{l}\text { Canalizar vía venosa para tomar muestras para laboratorio y para administrar líquidos } \\
\text { vía parenteral si es necesario. }\end{array}$ \\
\hline $\begin{array}{l}\text { Vigile la } \\
\text { respiración }\end{array}$ & $\begin{array}{l}\text { Vigilar la respiración. Si ésta se encuentra deprimida debe administrarse oxígeno } \\
\text { humidificado mediante catéter nasal a un flujo de } 4 \text { a } 6 \text { I/min., o aplicar ventilación } \\
\text { pulmonar asistida. } \\
\text { En fallos respiratorios severos, debe mantenerse la ventilación mecánica pulmonar } \\
\text { durante varios días. } \\
\text { La mejoría clínica y la concentración de los gases arteriales, son algunos parámetros } \\
\text { útiles para determinar cuándo retirar el ventilador. }\end{array}$ \\
\hline $\begin{array}{l}\text { Ponga sonda } \\
\text { nasogástrica }\end{array}$ & $\begin{array}{l}\text { En los casos de intoxicación por ingestión se debe colocar sonda nasogástrica y } \\
\text { realizar lavado gástrico con solución de bicarbonato de sodio al 5\% hasta que no se } \\
\text { perciba el olor del tóxico. }\end{array}$ \\
\hline $\begin{array}{l}\text { Administre un } \\
\text { catártico }\end{array}$ & $\begin{array}{l}\text { Administrar carbón activado junto con un catártico. Para adultos y niños mayores de } 12 \\
\text { años se recomienda suministrar de } 50 \text { a } 100 \text { gramos de carbón activado en } 300 \text { a } 800 \\
\text { ml de agua. Para niños menores de } 12 \text { años, de } 15 \text { a } 30 \text { gramos en } 100 \text { a } 300 \mathrm{ml} \text { de } \\
\text { agua. } \\
\text { Como catárticos puede emplearse sulfato de magnesio de sodio, utilizando las } \\
\text { siguientes dosis: } \\
\text { En adultos y niños mayores de } 12 \text { años, administrar de } 20 \text { a } 30 \text { gramos y en menores, } \\
250 \text { mg/Kg de peso corporal, el citrato de magnesio en dosis de } 4 \mathrm{ml} / \mathrm{Kg} \text { de peso, hasta } \\
\text { un máximo de } 300 \mathrm{ml} \text {. }\end{array}$ \\
\hline Diuresis & Mantener una excreta urinaria adecuada \\
\hline
\end{tabular}




\begin{tabular}{|l|l|}
\hline Evite Emesis & $\begin{array}{l}\text { Administrar los líquidos lentamente para evitar la emesis o la regurgitación. En niños } \\
\text { luego de la catarsis debe vigilarse con especial cuidado los trastornos electrolíticos, } \\
\text { debe evitarse los catárticos a base de Magnesio en enfermedad renal y los catárticos } \\
\text { con sodio en falla cardíaca congestiva e hipertensión severa. } \\
\text { Si el paciente está consciente, puede administrársele el carbón activado y el catártico } \\
\text { por vía oral. El catártico debe indicarse cada } 4 \text { - } 6 \text { horas hasta que aparezca diarrea. } \\
\text { Recoger una muestra de vómito o del producto del avado gástrico inicial para análisis } \\
\text { químico. }\end{array}$ \\
\hline
\end{tabular}

Fuente: (Castro Valencia, 2015)

\section{Tratamiento}

En cuanto a las medidas específicas, se refiere a la administración del antídoto:

- Atropina: 0,01-0,05mg/Kg (niños) $1 \mathrm{a}$ $2 \mathrm{mg}$ (adultos) EV C/5-10min. durante 1 hora hasta que aparezcan signos de atropinización (rubor facial, midriasis, boca seca, taquicardia, desaparición de secreciones bronquiales y/o confusión mental con alucinaciones). Dependiendo de la evolución del cuadro se distancia la dosis progresivamente cada 30min., 45min., 60min. hasta omitirla.

- Otro método: diluir la atropina en solución salina o glucosada para titular la dosis calculada en infusión continua hasta obtener atropinización. Mientras que la vía venosa no esté disponible, la atropina será administrada por vía intramuscular, subcutánea, endotraqueal o intraosea (niños).

- Oximas: toxogonin (cloruro de obidoxima) $250 \mathrm{mg} / \mathrm{ml} \mathrm{EV}$, repetir $20 \mathrm{~min}$ después. Todos los pacientes deberán permanecer bajo observación estricta después de cesar la terapia con oximas. En caso de recaída de las manifestaciones clínicas indicaría la necesidad de proseguir (Cuasapaz Guama, 2017).

Las medidas generales están encaminadas a la realización de acciones efectivas del personal de enfermería en la atención del paciente intoxicado con organofosforados, y cuya vía de penetración al organismo es la ingesta, pero para este fin es necesario conocer la forma en que actúa el tóxico y la manera como antagonizar sus efectos. Ahora bien, una valoración inicial y rápida de la enfermera(o) debe identificar los siguientes signos y síntomas:

- Perturbación de la visión (miosis y visión borrosa)

- Incremento en las secreciones como epifora, sialorrea, diaforesis

- Bradicardia

- Disnea o bradipnea

- Nauseas, vómito, diarrea

- Aumento de los ruidos hidroaéreos

- Palidez, cianosis

- Fasciculaciones de músculos

- Debilidad muscular o parálisis

- Decremento del nivel de conciencia

- Actividad convulsiva (Cuasapaz Guama, 2017).

Es conveniente precisar que cuando el toxico penetra por la piel es necesario retirar la ropa y bañar al paciente. Se plantea entonces, que lo anteriormente expuesto, se logra siguiendo las siguientes medidas:

- Aspiración de las vías respiratorias para extraer secreciones ya que existe aumento de las mismas.

- Cateterización de vía periférica con catéter calibre grueso para: obtener muestra para hematimetría, gasometría, electrolitos, glucosa, nitrógeno úrico, y niveles de creatinina, medir la actividad de colinesterasa en los eritrocitos. 
- Administrar atropina

- Oxigenoterapia si hay cianosis para corregir hipoxia, evitando así la fibrilación como una reacción adversa a la atropina

- Mantener sonda nasogástrica calibre grueso para toma de muestra gástrica, lavado gástrico y administración de carbón activado

- Mantener sonda vesical para controlar diuresis

- Control de signos vitales horarios, ya que existe la posibilidad de surgimiento de hipotensión arterial y bradicardia, inclusive bloqueo cardíaco, además se puede producir alteraciones en la temperatura

- Posición adecuada thendelenburg para mejorar el riesgo a órganos vitales

- Auscultación cardio-pulmonar, se puede presentar estertores, taquipnea o esputos espumosos que denoten edema pulmonar

- Evaluación neurológica sobre todo el nivel de conciencia cada hora

- Valorar fasciculaciones y contracciones musculares (Cuasapaz Guama, 2017).

\section{Conclusión}

Como se ha podido leer en esta investigación, los componentes que tienen los plaguicidas pueden ser altamente tóxicos, en la mayoría de los casos los mayores porcentajes de intoxicación por estos productos se producen en los sectores agrícolas. Sin embargo, plaguicidas de uso regular en el hogar contra roedores y hasta mosquitos pueden ser igual de tóxicos y producir los mismos efectos que un plaguicida agrícola. La sintomatología cuando una persona ha sido expuesta a un plaguicida altamente toxico suele aparecer entre $1 / 2$ y 1 hora.

Una intoxicación por plaguicida puede ser mortal, independientemente de por cuál vía la persona haya tenido contacto con el mismo. Entre algunos de los síntomas que se pueden presentar, son dificultad respiratoria, disnea, irritación en ojos y piel, bradicar- dia, entre otros. Es por ello que la valoración inicial que se haga en el centro de salud por el personal médico más la información que pueda proporcionar los familiares, compañeros de trabajo, del intoxicado acerca del tipo de plaguicida puede ser determinante para el abordaje rápido en cuanto a la administración del tratamiento mas adecuado. El tratamiento va a depender de el grado de la intoxicación, sin embargo, entre los tipos de tratamiento esta el uso de Atropina y Oximinas, colocación de sondas nasogástricas y lavado gástricos, administración de carbón activado, entre otros.

\section{Bibliografía}

Aveiga Valdivieso, A. A. (2020). Aplicación de un protocolo de manejo de intoxicaciones agudas por plaguicidas. Area de emergencias del Hospital del Niño" Francisco Icaza Bustamante", 2010.

Barco, J. C., Omar, C., Puiguriguer , J., Coll, I., Barcelo, B., \& Castanyer, T. (2008). Revisión de las intoxicaciones graves por insecticidas organofosforados atendidas en un período de 11 años (1996-2006). Emergencias, 20(3), 207-211.

Castro Valencia, C. (2015). Propuesta de protocolo de atención de enfermería en pacientes con intoxicación por órgano fosforado en el Hospital de Tena enero-diciembre 2010.

Cerna Urrutia, L. E., \& Trigueros Jovel, J. (2015). Epidemiología y evolución clínica de pacientes intoxicados por plaguicidas atendidos en el Hospital Nacional San Rafael en el período de enero 2013 a junio 2014.

Conejo, M., Órnelas, R., \& de los Ángeles, M. (s.f). GUÍA PARA PREVENIR INTOXICACIÓN POR PLAGUICIDAS.

Cuasapaz Guama, V. L. (2017). Conocimientos de enfermería sobre intoxicaciones por organofosforado del Servicio de Emergencia Hospital General Ibarra.

Gutiérrez, M. (2010). Incidencia de intoxicación por plaguicidas en mujeres temporeras de las comunas de Nacimiento y Negrete, provincia de Bío Bío 2003. 

MERCIAL-COMPARTIRIGUAL 4.0 .

\section{CITAR ESTE ARTICULO:}

Díaz Pérez, A. A., Luzuriaga Amador, J. M., Monroy Pesantez, M. F., \& Vera Hinojosa, J. A. (2021). Manejo de emergencia en intoxicación por plaguicidas. RECIMUNDO, 5(2), 179-186. https://doi.org/10.26820/recimundo/5.(2). abril.2021.179-186 